\title{
Application of Quality Control Circle in Improving the Correct Implementation Rate of Multiple Drug Resistant Organism Isolation Measures in ICU Medical Personnel
}

\author{
Dongliang Xu*, Weitao Lv, Yixiang Liu, Ziqiang Yu, Yiting Feng, Jiaxing Tang \\ Intensive Care Unit, The First Affiliated Hospital of Jinan University, Guangzhou, China
}

Email address:

505041109@qq.com (Dongliang Xu)

${ }^{*}$ Corresponding author

To cite this article:

Dongliang Xu, Weitao Lv, Yixiang Liu, Ziqiang Yu, Yiting Feng, Jiaxing Tang. Application of Quality Control Circle in Improving the Correct Implementation Rate of Multiple Drug Resistant Organism Isolation Measures in ICU Medical Personnel. American Journal of Nursing Science. Vol. 9, No. 6, 2020, pp. 402-406. doi: 10.11648/j.ajns.20200906.12

Received: October 13, 2020; Accepted: October 22, 2020; Published: October 30, 2020

\begin{abstract}
Objective: Effective isolation management of multiple drug resistant organism (MDRO) patients is considered an important measure to prevent cross-infection. To reduce the spread of MDRO, there is a need to improve the correct implementation of isolation measures for intensive care unit (ICU) medical personnel. We will investigate the application of quality control circle (QCC) activities in improving the correct implementation rate of isolation measures for patients with MDRO in ICU medical personnel. Methods: A 10-member QCC activity group was established. All of the ICU medical personnel were taken as the research objects, including doctors, nurses, assistant nurses, rotation training personnel, external ICU consultants and cleaning personnel. The correct implementation of isolation measures for patients with MDRO infection were investigated and studied, which identified the problem areas and analyzed the main reasons. The quality improvement countermeasures were formulated and implemented in ICU medical personnel, while the results were compared with before and after the QCC activities. Results: After QCC activities, the correct implementation rate of isolation measures by ICU medical personnel for patients with MDRO infection increased from $57.7 \%$ to $82.4 \%$. The incidence of MDRO infection in ICU patients decreased from $9.64 \%$ to $3.77 \%$, with statistically significant differences $(P<0.05)$. Conclusion: The QCC activities improved the correct implementation rate of MDRO isolation measures by ICU medical personnel, standardizing the isolation management of MDRO patients, while the incidence of MDRO in ICU patients was also effectively reduced.
\end{abstract}

Keywords: Multiple Drug Resistant Organism, Quality Control Circle, Isolation Measures, Correct Implementation Rate

\section{Introduction}

Multiple drug resistant organism (MDRO) refers to bacteria that are resistant to three or more antimicrobial agents used in clinical practice, which have become an important pathogen of nosocomial infections [1,2]. At present, MDRO infection has become a serious problem threatening the safety of intensive care unit (ICU) patients [3]. Effective isolation management of MDRO patients is considered an important measure to prevent cross-infection and reduce the incidence of MDRO [4]. To prevent and control the hospital-based prevalence and outbreak of MDRO, the ICU medical personnel in isolation for MDRO infection prevention needs to strictly follow the "Technical Guidelines for the Prevention and Control of MDRO Infections" [5]. However, in fact, those regulations are not strictly enforced by ICU medical personnel in the implementation of the MDRO preventive isolation measures with poor compliance or other phenomena. The Quality Control Circle (QCC) is a quality management group, which is composed of staff from the same or similar or complementary workplaces who automatically form an organizational group with several people in one circle, maintain cooperation, brainpower, and solve quality management problems by applying seven techniques of Quality management according to certain activity procedures [6]. In recent years, QCC activities have been widely carried out and applied in nursing work, effectively promoting the continuous improvement of nursing quality and patient 
rehabilitation [7]. To prevent cross infection ICU patients, promote patients recovery process, improve patient satisfaction, it was established as the name of "Guardian Circle" team of QCC activities since February 2019 in our comprehensive ICU area. The theme was formulated as "improving the correct implementation rate of isolation measures for patients with MDRO in ICU medical personnel". After 10 months of continuous improvement, good results have been achieved, as reported below.

\section{Materials and Methods}

\subsection{General Information}

Our comprehensive ICU doctors, nurses, assistant nurses, rotation and training personnel, external consultants and cleaning personnel were taken as the research objects, including 52 medical staff, 4 assistant nurses, 10 rotation and training personnel, 4 external consultations and 2 cleaning personnel, with the age range from $22 \sim 59$ years old. Their work data, including patients' clinical data and medical records, were collected from August 2018 to January 2019 as the control group before the QCC activity, and from June 2019 to November 2019 as the observation group after the QCC activity.

\subsection{Methods}

\subsubsection{Set up a Quality Control Circle Group}

A total of 10 members QCC activity group was established. The head nurse served as the head of QCC group, and the department director nurse as the instructor. The circle members proposed the circle name through brainstorming, and finally selected "Guardian Circle" as the circle name by voting.

\subsubsection{Made an Activity Plan}

The activity was scheduled from February 2019 to June 2019. The quality control Circle team made an activity schedule from collecting data, identifying problems, analyzing causes, demonstrating important causes, formulating countermeasures, implementing measures, checking feedback, and making continuous improvement. The activity group will hold the circle activity report twice a month, set the target by stages, implement the effect evaluation of nursing measures, summarize experience and further standardize.

\subsubsection{Current Situation Investigation}

With the assistance of the Nosocomial Infection Department, the investigation team will collect work data from August 2018 to January 2019, including clinical data and medical records of patients, investigate the current situation of MDRO infection in our department, and analyze the problems existing in the implementation of isolation measures for medical staff in the MDRO. With reference to the Ministry of Health "Technical Guidelines for the Prevention and Control of MDRO Infections", the self-designed questionnaire was used for survey ICU medical personnel of MDRO infection and the related knowledge about isolation control situation [8, 9]. The results showed that the medical staff in the ICU of MDRO isolation measures witting rate was $80.56 \%$, and the corresponding measures were enforced only $69.44 \%$, which was far from the requirements of the guidelines.

\subsubsection{Analysis of Causes}

For the ICU medical personnel of MDRO isolation measures enforced low status, group members analyzed of the main reasons by "fishbone diagram" method, including: (1) the medical staff had not enough of MDRO isolation measures of prevention and control knowledge: part of the low qualification of doctors and nurses has been ignored in MDRO risk factors, diagnosis standard, MDRO infection isolation measures and other basic knowledge, with also insufficient understanding of the consequences of MDRO. (2) Low hand hygiene compliance: the standard of seven-step washing hand was not well mastered, the timing and indication of hand hygiene were not proficient, the heavy workload, hand washing conditions, facilities and other factors lead to the low hand hygiene implementation rate of medical staff [10]. (3) Low implementation rate of isolation measures: medical staff were not aware of disinfection and isolation, and the utilization rate of protective equipment was low; Rotation and the large number of foreign personnel, the flow of the hospital environment was not enough to understand; The implementation rate of isolation protection was low due to the unreasonable placement of protective equipment in different zones. (4) Unqualified environmental and disinfection measures: the unreasonable distribution of beds in patients with multi-drug resistant bacteria leads to cross-infection; Frequent movement of medical personnel during operation causes air pollution; Poor compliance of cleaning and disinfection procedures of cleaning personnel is the result of unqualified disinfection of environmental surface; Lack of solid knowledge of garbage classification, unreasonable setting of clean and polluted areas and so on cause unqualified medical waste treatment.

\subsubsection{Implementation Activities}

Aiming at main problems above, formulate the corresponding improvement measures were: (1) to develop Standard Operating Procedure (SOP) [11]: reference the Ministry of Health "Technical Guidelines for the Prevention and Control of MDRO Infections" and the "Hospital Infection Prevention and Control of MDRO China Expert Consensus" to develop SOP for the prevention and control of MDRO in ICU. After discussion and revision by the department of infectious diseases and the committee of hospital Infection management, a practical and operational SOP system has been formed and strictly implemented. (2) to develop training programs: based on the Ministry of Health "Technical Guidelines for the Prevention and Control of MDRO Infections", the "Medical Staff Hand Hygiene Standard" [12] and the "Medical Institutions Technical Standard for Disinfection", a training program for ICU medical personnel on MDRO isolation measures were redacted, training content including hand hygiene norms, proper use of personal 
protective equipment, correct method of disinfection of air and environment surface, the treatment system of medical waste, etc. QCC insider member of division of labor, according to different objects for personalized training programs, this department staff and school cooperation and foreign education theory and operation training for rotary personnel, and production of medical staff in contact with multi-resistant bacteria of analog video, let trainees in the video was inspired, assessment standard rear can contact with multi-resistant bacteria. Hold monthly circle activities summary feedback report, summarize the shortcomings, and continue to improve. (3) Strengthen hand hygiene management: learn and firmly grasp hand washing and hand disinfection indications, master standard hand washing methods and assessment, and equip with enough quick hand washing disinfectant to improve hand hygiene compliance. (4) Strict isolation measures: isolate the MDRO infected patient in a single room, and hang warning signs beside the bed. Adequate amount of protective equipment (isolation clothes, gloves, masks, shoe covers, etc.) shall be installed at designated places for convenient use. Special persons shall use the special bed counters for articles. Schedule care for patients with a high degree of suspected or confirmed MDRO infection until the end. (5) Strengthen cleaning and disinfection of articles: patients' beds (including bed frames) and bedside tables should be thoroughly cleaned and disinfected every day. General medical instruments such as stethoscope, thermometer or sphygmomanometer for special use, soak, wipe every day, and cannot be dedicated items such as wheelchair, flat car, disinfection after each use. Medical waste should be properly disposed of and managed in accordance with relevant regulations.

\subsection{Effect Evaluation}

Comparison between before and after QCC activity, ICU medical personnel's correct implementation of various isolation measures for patients with MDRO infection, including hand hygiene standards, proper use of personal protective equipment, qualified disinfection of air and environment surfaces, and proper disposal of medical waste, etc. The incidence of MDRO infection in ICU patients before and after QCC activity was calculated.

\subsection{Statistical Methods}

SPSS21.0 statistical software was used for statistical analysis. Measurement data were expressed as mean \pm standard deviation $(\mathrm{x} \pm \mathrm{s})$, and comparison between groups was performed by $t$ test. Statistical data were expressed in terms of frequency and percentage (\%), and $P<0.05$ was considered statistically significant when chi-square test was used.

\section{Results}

\subsection{Implementation of Isolation Measures}

Comparison of the implementation of various isolation measures for MDRO patients by ICU medical personnel before and after QCC activities was shown in Table 1. The pass rate of all measures was significantly improved, and the difference was statistically significant $(P<0.05)$. After the implementation of the activity, the qualified rate of ICU medical personnel in implementing isolation measures for MDRO patients increased from $69.44 \%$ to $90.00 \%(P<0.05)$.

Table 1. Comparison of the implementation of isolation measures for ICU medical personnel before and after QCC activity Cases (\%).

\begin{tabular}{llll}
\hline Projects & Before QCC (n=72) & After QCC (n=70) & $\chi^{2}$ \\
\hline Hand hygiene qualified & $55(76.39)$ & $67(95.71)$ & 9.416 \\
Used personal protective equipment correctly & $54(75.00)$ & $66(94.29)$ & 8.664 \\
Air environmental surface disinfection qualified & $52(72.22)$ & $65(92.86)$ & 10.418 \\
Medical waste disposal qualified & $56(77.78)$ & $65(92.86)$ & 0.003 \\
All qualified & $50(69.44)$ & $63(90.00)$ & 0.001 \\
\hline
\end{tabular}

\subsection{Comparison of MDRO Infection Rates}

The infection rates of the control group before the QCC activity (August $2018 \sim$ January 2019) and the observation group after the activity (June $2019 \sim$ November 2019) were recorded, as shown in Table 2. After QCC activity, the incidence of MDRO infection in ICU patients decreased from $10.47 \%$ to $4.05 \%$, with a statistically significant difference $(P$ $<0.05)$.

Table 2. Comparison of MDRO infection rates before and after QCC activity (Cases).

\begin{tabular}{llll}
\hline Projects & Cases & MDRO Infection cases & MDRO Infection rates \\
\hline Before QCC activity & 382 & 40 & $10.47 \%$ \\
After QCC activity & 395 & 16 & $4.05 \%$ \\
$\chi^{2}$ & & & 11.970 \\
$P$ & & & 0.001 \\
\hline
\end{tabular}

\section{Discuss}

The continuous emergence of MDRO has become a serious problem threatening the safety of ICU patients, especially causing nosocomial infections, resulting in the increase of fatality rate of patients and the sharp rise of medical costs [13]. On May 26, 2019, the General Office of the National Health Commission issued the "Notice on Further Strengthening the Work of Infection Prevention and Control in Medical 
Institutions", which explicitly requires the strict implementation of prevention and control measures for MDRO infections [14]. In this study, QCC activity techniques were applied to improve the correct implementation rate of isolation measures for patients with MDRO in ICU medical personnel. There were intervened in hand hygiene, using of personal protective equipment, disinfection of air and environmental surfaces, and treatment of medical waste, etc. At the same time, they were taken as the action guidelines for the prevention and control of MDRO in ICU, which referenced the Ministry of Health "Technical Guidelines for the Prevention and Control of MDRO Infections" and the "Hospital Infection Prevention and Control of MDRO China Expert Consensus" [15]. The correct implementation rate of isolation measures could be effectively improved through continuously strengthening and standardizing the isolation operation of medical personnel. They were trained in batches, including doctors, nurses, assistant nurses, rotation and training personnel, external consultants and cleaning personnel, and follow up the training effect and field guidance to ensure the implementation of each link. After the implementation of QCC, the qualified rate of the implementation of various isolation measures and the overall qualified rate of ICU medical personnel were significantly improved. While the incidence of MDRO infection in ICU patients decreased from $10.47 \%$ to $4.05 \%$.

Due to tracheotomy, long-term bed rest, and various treatments, the medical environment of comprehensive ICU patients was often cross-polluted with MDRO [16]. The ICU medical personnel correct execution of isolation measures directly affected the transmission and infection of MDRO in ICU patients. Through the QCC program, we realized that it was important to raise awareness of prevention and control of MDRO among healthcare professionals. In medical staff, on the basis of full understanding the harm of MDRO infection, according to the concrete problem formulation corresponding isolation measures, and constantly improve and perfect in the practical action, there were make ICU medical personnel in MDRO correctly perform the isolation measures in the prevention and control of infection. On this basis, further standardized isolation measures to form a practical standard operating procedure, which could effectively prevent and control the transmission of MDRO in the hospital, and it was worthy for clinical promotion and application.

\section{Conclusion}

According to the concrete problem formulation corresponding isolation measures, and constantly improving and perfecting in the practical action, the QCC project promoted the ICU medical personnel to correctly implement all isolation measures in the prevention and control of MDRO infection, effectively prevented and controlled the transmission of MDRO in the hospital. At the same time, QCC activities could effectively improve the self-discipline and initiative of medical staff, and unconsciously develop the correct implementation habit of isolation measures for patients with MDRO infection, which the rate of correct implementation has been significantly improved. The application of the QCC effectively prevented cross infection of ICU patients, promoted patients recovery process, improved patient satisfaction, so it deserved application and promotion in clinical work.

\section{References}

[1] Siljander M, Sobh A, Baker K C, et al. Multi-Drug Resistant Organisms in the Setting of Periprosthetic Joint Infection Diagnosis, Prevention and Treatment [J]. Journal of Arthroplasty, 2017, 33 (1): 185-194.

[2] Labricciosa F M, Massimo S, Abbo L M, et al. Epidemiology and Risk Factors for Isolation of Multi-Drug-Resistant Organisms in Patients with Complicated Intra-Abdominal Infections.[J]. Surgical Infections, 2018, 19 (3): 264-272.

[3] Ying X U, Bing H, Jie Y, et al. Clinical pharmaceutical care of the infection of multiple drug resistant bacteria in ICU [J]. Shanghai Medical \& Pharmaceutical Journal, 2019, 40 (03): 49-51.

[4] Von Cube M K, Timsit J F, Sommer H, et al. Relative risk and population-attributable fraction of ICU death caused by susceptible and resistant Pseudomonas aeruginosa ventilator-associated pneumonia: a competing risks approach to investigate the OUTCOMEREA database.[J]. Intensive Care Medicine, 2018, 44 (7): 1177-1179.

[5] Yun Z, Ning-Ning X, Yi Z, et al. Value of application of process reengineering in management of multidrug-resistant organism infections [J]. Chinese Journal of Nosocomiology, 2016, 26 (01): 207-208+220.

[6] Guichun W, Yehong K, Xiuqing L I. The effects of quality control circle management for nurses in increasing specification rate for nurses in application of Ecg monitor [J]. Heb Medical Journal, 2019, 41 (02): 318-320.

[7] Yan-Fang W U. Effect of Quality Control Circle Management of PDCA Cycle on Treatment Compliance and Quality of Life in Postoperative Patients with Breast Cancer [J]. Clinical Research, 2019, 27 (06): 173-175.

[8] The Royal College of Physicians Clinical Advisory Group on Healthcare Associated Infections. Guidelines for the prevention and control of multi-drug resistant organisms (MDRO) excluding MRSA in the healthcare setting [M]// Theory and practice of animal taxonomy /. Oxford \& IBH Publishing Co. 2012.

[9] Hee P S. Management of multi-drug resistant organisms in healthcare settings [J]. Journal of the Korean Medical Association, 2018, 61 (1): 26.

[10] Belela-Anacleto A S C, Kusahara D M, Peterlini, Maria Angélica $\mathrm{S}$, et al. Hand hygiene compliance and behavioural determinants in a paediatric intensive care unit: An observational study $[\mathrm{J}]$. Australian Critical Care Official Journal of the Confederation of Australian Critical Care Nurses, 2018, 32 (1): 21-27.

[11] Cui L, Ye-Dan W, Sai-Duan L, et al. Application effect of standard operation procedure for patients undergoing operation [J]. Hainan Medical Journal, 2018, 29 (10): 1476-1478. 
[12] Soon L S, Jeong P S, Joo C M, et al. Improved Hand Hygiene Compliance is Associated with the Change of Perception toward Hand Hygiene among Medical Personnel [J]. Infection \& Chemotherapy, 2014, 46 (3): 165-171.

[13] Min Z, Qiang S. Study on the Control Measures of MDRO Transmission in ICU Based on Markov Process [M]// Smart Service Systems, Operations Management, and Analytics. 2020.

[14] Jinzhu W, Yunxia F, Hui S, et al. Monitoring and analysis of disinfection effect of medical institutions in Hedong District of Tianjin in 2014-2018 [J]. Journal of Medical Pest Control, 2020, 36 (02): 197-198.

[15] Wilson A P R, Livermore D M, Otter J A, et al. Prevention and control of multi-drug-resistant Gram-negative bacteria: recommendations from a Joint Working Party [J]. Journal of Hospital Infection, 2016, 92: S1-S44.

[16] Dan W, Dan Z, Hong C, et al. Multidrug-resistant organism healthcare-associated infection and economic burden in general intensive care unit patients $[\mathrm{J}]$. Chinese Journal of Infection Control, 2019, 18 (07): 648-653.

\section{Biography}

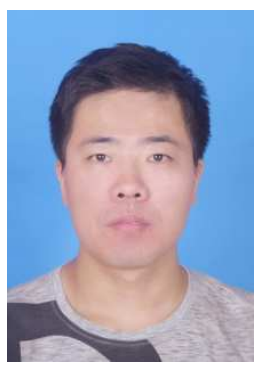

Dongliang Xu, Male, Bachelor, Nurse practitioner, Dedicated to Intensive Care Unit (ICU) nursing and ICU management. 